\title{
Squeezing-enhanced phase-shift-keyed binary communication in noisy channels
}

\author{
Giovanni Chesi, ${ }^{1}$ Stefano Olivares,,${ }^{2,3, *}$ and Matteo G. A. Paris ${ }^{2,3}$ \\ ${ }^{1}$ Department of Science and High Technology, University of Insubria, Via Valleggio 11, I-22100 Como, Italy \\ ${ }^{2}$ Quantum Technology Lab, Dipartimento di Fisica "Aldo Pontremoli”, Università degli Studi di Milano, I-20133 Milano, Italy \\ ${ }^{3}$ INFN, Sezione di Milano, I-20133 Milano, Italy
}

(Received 23 October 2017; published 15 March 2018)

\begin{abstract}
We address binary phase-shift-keyed communication channels based on Gaussian states and prove that squeezing improves state discrimination at fixed energy of the channel, and also in the presence of phase diffusion. We then assess performances of homodyne detection against the ultimate quantum limits to discrimination and show that homodyning achieves optimality in the large-noise regime. Finally, we consider noise in the preparation of the seed signal (before phase encoding) and show that, also in this case, squeezing may improve state discrimination in realistic conditions.
\end{abstract}

DOI: 10.1103/PhysRevA.97.032315

\section{INTRODUCTION}

Quantum hypothesis testing addresses the discrimination of nonorthogonal preparations of a quantum system. As such, it has attracted attention from a fundamental point of view [1-9], and represents a central tool for the design and development of quantum communication channels. In particular, much attention has been payed to quantum binary discrimination, which already shows a rich quantum phenomenology amenable to analytic investigations and fosters promising perspectives in a number of challenging applications, such as quantum communication $[10,11]$ and quantum cryptography [12]. In particular, quantum-optical implementations of state discrimination have been investigated [13-15], with the focus on information carried by coherent states [16,17], although also the use of squeezing has been explored to some extent $[18,19]$.

In quantum binary communication, one has to discriminate between two quantum signals. Assuming a pure preparation, i.e., ignoring the presence of noise, we may denote the two states by $\left|\psi_{1}\right\rangle$ and $\left|\psi_{2}\right\rangle$. The minimum error probability is not vanishing for nonorthogonal states and is given by the so-called Helstrom bound [2] (throughout the paper we assume equal prior probability for the two signals):

$$
P^{(\min )}\left(\left|\psi_{1}\right\rangle,\left|\psi_{2}\right\rangle\right)=\frac{1}{2}\left(1-\sqrt{1-\left|\left\langle\psi_{1} \mid \psi_{2}\right\rangle\right|^{2}}\right) .
$$

In quantum communication channels the two states are often obtained from a seed state $\left|\psi_{0}\right\rangle$ through a suitable unitary transformation. In coherent-state communication, the quantum signal $\left|\psi_{k}\right\rangle, k=1,2$, is obtained by applying a displacement operation to the vacuum state $|0\rangle$; namely,

$$
\left|\psi_{k}\right\rangle=D\left[(-1)^{k} \alpha\right]|0\rangle, \quad(k=1,2),
$$

where $D(\alpha)=\exp \left(\alpha a^{\dagger}-\alpha^{*} a\right),\left[a, a^{\dagger}\right]=\mathbb{I}$ and we can assume $\alpha \in \mathbb{R}, \alpha>0$. Since the difference between the two states is an overall $\pi$ phase, we refer to this encoding as phase-shift keying (PSK). This channel has been thoroughly investigated because

\footnotetext{
*stefano.olivares@ fisica.unimi.it
}

of the peculiar properties of coherent states: they can be easily generated and manipulated and their phase properties are not affected by losses (only their amplitude is reduced). Therefore, they can travel for long distances preserving their coherence properties $[11,20]$.

The search for optimal receivers, i.e., detection schemes and strategies able to discriminate between the two coherent states reaching the corresponding Helstrom bound, has eventually led to the so-called Kennedy receiver [21] and Dolinar receiver [22]. These are both based on the interference of the signals with a known reference, and on on-off photodetectors, able to check the presence or absence of light. The Dolinar receiver also exploits a feedback mechanism in order to achieve the Helstrom bound (it requires, however, many copies of the same input state in order to implement the feedback). These kinds of detectors require a precise phase reference control and in the presence of phase fluctuations their performances are drastically reduced [23]. Nevertheless, it has been shown that, in the presence of phase noise, a receiver based on homodyne detection [24] allows us to approach optimality [25].

Recently, coherent-state encoding and homodyne detection have been used to establish ground-satellite links [11], whereas squeezed states have been suggested to improve quantum key distribution [26]. Motivated by these results, here we consider a binary communication channel in which the seed $\left|\psi_{0}\right\rangle$ is a squeezed vacuum $|r\rangle=S(r)|0\rangle$ where $S(r)=$ $\exp \left\{-\frac{1}{2}\left[r\left(a^{\dagger}\right)^{2}-r^{*} a^{2}\right]\right\}$ is the squeezing operator. Without lack of generality we assume $r \in \mathbb{R}$. In this case the input states are

$$
\left|\psi_{1}\right\rangle=|-\alpha, r\rangle \text { and }\left|\psi_{2}\right\rangle=|\alpha, r\rangle,
$$

where we introduced the displaced squeezed state (DSS)

$$
|\alpha, r\rangle=D(\alpha) S(r)|0\rangle .
$$

In the following, we first address the Helstrom bound for two DSSs and compare the results with those obtained with coherent states and/or using homodyne detection. We then investigate the effect of phase diffusion on the discrimination and compare our results with the ultimate quantum limits, i.e., 
the corresponding Helstrom bound. Finally, we analyze the effect of losses, resulting in a reduced purity of the seed state.

\section{DISCRIMINATION BETWEEN DISPLACED-SQUEEZED STATES}

Let us start by investigating the ultimate performances of DSSs with respect to the coherent-state ones in the absence of noise and with optimal detection. This corresponds to evaluating the Helstrom bound for the input states given in Eqs. (3). For the sake of simplicity, we can assume $\alpha, r \in \mathbb{R}$, with $\alpha, r>0$. The corresponding Helstrom bound can be easily calculated from Eq. (1) and reads

$$
\begin{aligned}
P^{(\min )}(r, \alpha) & =\frac{1}{2}\left(1-\sqrt{1-|\langle\alpha, r \mid-\alpha, r\rangle|^{2}}\right) \\
& =\frac{1}{2}\left[1-\sqrt{1-\exp \left(-4 \alpha^{2} e^{2 r}\right)}\right] .
\end{aligned}
$$

It is clear that, for fixed coherent amplitude, squeezing always allows us to improve the discrimination. However, the squeezing operation is adding energy to the coherent states, resulting in a comparison between two encodings with different total energy. A better comparison may be obtained by fixing the total energy used in the communication channel which is usually set by physical constraints. For the coherent-state channel based on $| \pm \alpha\rangle$ we have the following average number of photons: $N_{\mathrm{CS}}=| \pm \alpha|^{2}$, whereas, if we employ DSSs $| \pm \alpha, r\rangle$, the energy is given by $N_{\mathrm{DSS}}=|\alpha|^{2}+N_{\mathrm{sq}}$, where $N_{\mathrm{sq}}=\sinh ^{2}(r)$ is the average number of photons added by the squeezing process. If we impose the constraint on the energy, we can clearly see that the more the state is squeezed the less is displaced and vice versa; hence, the discrimination problem becomes nontrivial.

Our aim is to find the regimes in which squeezing can be a useful resource for the discrimination. Therefore, in the following we are going to study the error probability as a function of the introduced amount of squeezing (and at fixed total energy). Given the channel energy $N$ and the fraction of squeezing:

$$
\beta \equiv \frac{N_{\mathrm{sq}}}{N}=\frac{\sinh ^{2}(r)}{N},
$$

we can rewrite Eq. (5) expressed in terms of $\beta$ and $N$, namely:

$$
\begin{aligned}
P^{(\min )}(\beta, N)= & \frac{1}{2}-\frac{1}{2}\{1-\exp [-4 N(1-\beta) \\
& \times(1+2 N \beta+2 \sqrt{N \beta(1+N \beta)})]\}^{\frac{1}{2}} .
\end{aligned}
$$

We plot the behavior of $P^{(\min )}(\beta, N)$ in the left panel of Fig. 1. We can find an interval of $\beta$ values such that the error probability of a pair of DSSs is smaller than the corresponding coherent case. Then two critical values for $\beta$ are naturally identified: a threshold value $\beta_{\mathrm{th}}(N)$ and an optimum value $\beta_{\text {opt }}$. For $0<\beta<\beta_{\text {th }}$, DSSs achieve better performances with respect to coherent states and, in particular, it is possible to minimize the error probability by choosing a suitable squeezing fraction $\left(\beta_{\mathrm{opt}}\right)$. These quantities can be obtained analytically and read

$$
\beta_{\mathrm{th}}(N)=\frac{4 N}{4 N+1} \text { and } \beta_{\mathrm{opt}}(N)=\frac{N}{2 N+1},
$$

respectively. It is straightforward to see that, in the limit $N \gg 1$, one finds $\beta_{\text {th }} \rightarrow 1$ and $\beta_{\text {opt }} \rightarrow 1 / 2$. In this limit,
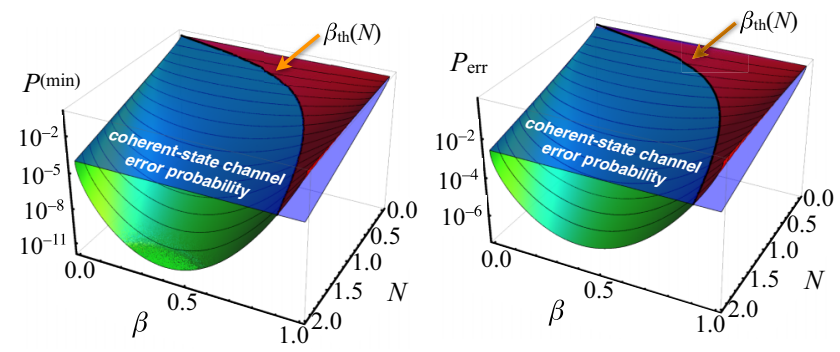

FIG. 1. (left) The Helstrom bound (7) as a function of the squeezing fraction $\beta$ and of the channel energy $N$. We also plot the plane corresponding to the Helstrom bound for coherent states. The solid line corresponds to the threshold $\beta_{\mathrm{th}}(N)$ : given the total energy $N$, if $\beta<\beta_{\mathrm{th}}(N)$ the use of DSSs outperforms coherent states. (right) The homodyne error probability $P_{\text {err }}$ of Eq. (12) as a function of $\beta$ for different values of the channel energy $N$. The behavior is qualitatively similar to the Helstrom bound reported in the left panel (see, however, the different scaling). We also plot the plane corresponding to the minimum error probability achievable by using only coherent states and homodyne detection.

the best strategy is to use half of the channel energy for squeezing. Moreover, we have that, for coherent states $(\beta=$ $0), P_{\mathrm{CS}}^{(\min )}=P^{(\min )}(0, N)=\frac{1}{2}[1-\sqrt{1-\exp (-4 N)}]$, whereas for DSSs with $\beta=\beta_{\text {opt }}$ we find $P_{\mathrm{DSS}}^{(\min )}=P^{(\mathrm{min})}\left(\beta_{\mathrm{opt}}, N\right)=$ $\frac{1}{2}\{1-\sqrt{1-\exp [-4 N(N+1)]}\}$. Thus, in the limit $N \gg 1$ the corresponding minimum error probabilities are

$$
\begin{aligned}
& P_{\mathrm{CS}}^{(\min )} \rightarrow \frac{1}{4} \exp (-4 N), \\
& P_{\mathrm{DSS}}^{(\min )} \rightarrow \frac{1}{4} \exp [-4 N(N+1)] \sim \frac{1}{4} \exp \left(-4 N^{2}\right),
\end{aligned}
$$

and the clear advantage can be also highlighted by considering the following ratio:

$$
\frac{P_{\mathrm{CS}}^{(\min )}(0, N)-P_{\mathrm{DSS}}^{(\min )}\left(\beta_{\mathrm{opt}}, N\right)}{P_{\mathrm{CS}}^{(\min )}(0, N)} \rightarrow 1-\exp \left(-4 N^{2}\right) .
$$

Let us now turn our attention to feasible measurements that we can perform on our optical states in order to discriminate between them. It is well known that, in the absence of noise and in the case of just two phase-shifted coherent states, there exist methods based on photon-number resolving detectors which allow one to approach the Helstrom bound [21,22,27,28]. Other proposals are based on an active detection stage; namely, a squeezing operation is applied to the signal before the detection [19].

However, one has to take into account noise to describe a realistic channel. Since we chose to employ a PSK channel, information is encoded on phase, so that the most detrimental kind of noise affecting our channel is phase noise. It has been shown [25] that homodyne detection is robust against phase noise; in particular, in the limit of large noise it beats the performance of a Kennedy receiver for every value of $N$ and achieves the Helstrom bound. These results lead us to consider homodyne detection in order to discriminate DSSs. On the one hand, this is a standard and well-established technique when dealing with continuous-variable optical systems, which is also promising in view of coherent-state communication 
with satellites [11]. On the other hand, the homodyne detection strategy has been proven to approach the Helstrom bound when phase noise affects the signal [25,29], which is the scenario we discuss in the next sections.

Homodyne detection allows us to measure the quadrature operator $x_{\theta} \equiv a e^{-i \theta}+a^{\dagger} e^{i \theta}$ of the input field [30], where $\left[a, a^{\dagger}\right]=\mathbb{I}$ and $\theta$ is the phase of the quadrature. Since we assumed real displacement amplitude and squeezing, we can focus on the measurement of $x_{0}$. As usual the two inputs $\mid \pm$ $\alpha, r\rangle$, with $\alpha, r>0$, are sent to the receiver, which measures $x$ and obtains the outcome $x \in \mathbb{R}$ from the homodyne detection. To discriminate between the two states, shot by shot, the receiver uses the following strategy:

$$
\left\{\begin{array}{l}
x \geqslant 0 \quad \Rightarrow \quad|0\rangle \equiv|\alpha, r\rangle, \\
x<0 \quad \Rightarrow \quad|1\rangle \equiv|-\alpha, r\rangle .
\end{array}\right.
$$

The error probability associated with this strategy writes

$$
P_{\text {err }}=\frac{1}{2}[p(x \geqslant 0 \mid 1)+p(x<0 \mid 0)]=p(x \geqslant 0 \mid 1),
$$

where we used $p(x<0 \mid 0)=p(x \geqslant 0 \mid 1)$. The conditional probabilities appearing in Eq. (12) are defined as

$$
p(x \geqslant 0 \mid 1)=\int_{0}^{+\infty} d x p^{(\mathrm{HD})}(x ; \pm \alpha, r)
$$

where $p^{(\mathrm{HD})}(x ; \pm \alpha, r)$ is the homodyne probability, which reads

$$
\begin{aligned}
p^{(\mathrm{HD})}(x ; \pm \alpha, r) & \left.\left.\equiv\right|_{0}\langle x \mid \pm \alpha, r\rangle\right|^{2} \\
& =\frac{1}{\sqrt{2 \pi e^{-2 r}}} \exp \left\{-\frac{[(x \pm 2 \alpha)]^{2}}{2 e^{-2 r}}\right\},
\end{aligned}
$$

with $x_{\theta}|x\rangle_{\theta}=x|x\rangle_{\theta}$ or, in terms of the squeezing fraction $\beta$ and the total number $N$ of photons, as

$$
p^{(\mathrm{HD})}(x ; \beta, N)=\frac{\exp \left\{-\frac{[x \pm 2 \sqrt{N(1-\beta)}]^{2}}{2 \Sigma^{2}}\right\}}{\sqrt{2 \pi \Sigma^{2}}},
$$

with $\Sigma=\Sigma(\beta, N)=(\sqrt{N \beta}+\sqrt{1+N \beta})^{-1}$. If we consider the regime $N \gg 1$, we obtain the following expressions of the error probabilities for coherent states $(\beta=0)$ :

$$
P_{\mathrm{err}, \mathrm{CS}} \rightarrow \frac{1}{4} \sqrt{\frac{2}{\pi}} \frac{e^{-2 N}}{\sqrt{N}}
$$

and for DSSs,

$$
P_{\text {err, DSS }} \rightarrow \frac{1}{4} \sqrt{\frac{2}{\pi}} \frac{e^{-2 N^{2}}}{N},
$$

respectively, where, in the latter we used $\beta=\beta_{\text {opt }}$ given in Eqs. (8). It is interesting to note that we find a dependence on $N$ for coherent states and on $N^{2}$ for the DDSs, as we obtained for the Helstrom bound in Eqs. (9) but, now, with a different scaling.

In the right panel of Fig. 1 we show $P_{\text {err }}$ as a function of the squeezing fraction $\beta$ and $N$. The plot well illustrates two features of the detection strategy. On the one hand, PSK based on DSSs outperforms the corresponding coherent protocol as far as the squeezing fraction is below a threshold value, which is the same minimizing the Helstrom bound i.e., that given in Eqs. (8). Notice that, being the encoding on pure states, the same working regime is also maximizing the mutual information between the sender and the receiver [9]. On the other hand, upon comparing the homodyne error probability with the ultimate bound of the left panel, we see that homodyne detection does not implement the optimum receiver, since the error probability is far from approaching the Helstrom bound. At the same time, it is known that homodyne performances with coherent encoding are useful in the presence of phase noise and, in particular, phase diffusion $[25,31]$. Therefore, in the following, we are going to investigate whether the use of squeezing can further improve the discrimination between the two signals when phase noise affects their propagation.

\section{DISCRIMINATION IN THE PRESENCE OF PHASE NOISE}

The effect of phase diffusion on a single-mode state can be described by the the following master equation [31,32]:

$$
\dot{\rho}(t)=\Gamma \mathcal{L}\left[a^{\dagger} a\right] \rho(t),
$$

where $\rho(t)$ is the density operator of the system, $\mathcal{L}[O] \rho=$ $2 O \rho O^{\dagger}-O^{\dagger} O \rho-\rho O^{\dagger} O$, and $\Gamma$ is the diffusion parameter. Simple calculations provide the following solution:

$$
\rho(t)=\sum_{n, m} \rho_{n, m}(t)|n\rangle\left\langle m\left|=\sum_{n, m} e^{-(n-m)^{2} \Delta} \rho_{n, m}(0)\right| n\right\rangle\langle m|,
$$

where $\rho_{n, m}(t)=\langle n|\rho(t)| m\rangle$ and $\Delta \equiv \Gamma t$.

Equation (19) can be rewritten in the following form:

$$
\rho(t)=\int_{-\infty}^{+\infty} d \phi \frac{\exp \left(-\frac{\phi^{2}}{2 \sigma^{2}}\right)}{\sqrt{2 \pi \sigma^{2}}} U_{\phi} \rho(0) U_{\phi}^{\dagger}
$$

where we introduced the phase shift operator $U_{\phi}=e^{-i \phi a^{\dagger} a}$, $\sigma^{2}=2 \Gamma t=2 \Delta$, and $\rho(0)=| \pm \alpha, r\rangle\langle \pm \alpha, r\rangle \mid$. From now on we refer to $\sigma$ as to the noise parameter.

Since $U_{\phi}| \pm \alpha, r\rangle=\left| \pm \alpha e^{-i \phi}, r e^{-i 2 \phi}\right\rangle$, one can easily calculate the error probability after the phase diffusion process starting from the homodyne probabilities:

$$
\begin{aligned}
& p_{\sigma}^{(\mathrm{HD})}(x ; \pm \alpha, r) \\
& \quad=\left.\left.\int_{-\infty}^{+\infty} d \phi \frac{\exp \left(-\frac{\phi^{2}}{2 \sigma^{2}}\right)}{\sqrt{2 \pi \sigma^{2}}}\right|_{0}\left\langle x \mid \pm \alpha e^{-i \phi}, r e^{-i \phi}\right\rangle\right|^{2} .
\end{aligned}
$$

In Fig. 2 (left panel) we plot the resulting error probability $P_{\text {err }}(\beta, N ; \sigma)$ as a function of $\beta$ and $N$ for different values of the noise parameter $\sigma$ : as one may expect, the error probability increases but, nevertheless, the DSSs perform better than the coherent states when $\beta$ is below a new threshold $\beta_{\mathrm{th}}(N, \sigma)$ that now depends on both $N$ and the noise parameter $\sigma$, as shown in the right panel of Fig. 2.

\section{A. Comparison with the ultimate bounds}

Here we compare the results obtained in the previous section with the minimum error probability given by the Helstrom bound in the presence of phase noise. Given two mixed states $\rho_{1}$ and $\rho_{2}$, the Helstrom bound reads [2]

$$
P^{(\min )}\left(\rho_{1}, \rho_{2}\right)=\frac{1}{2}\left[1-D\left(\rho_{1}, \rho_{2}\right)\right],
$$



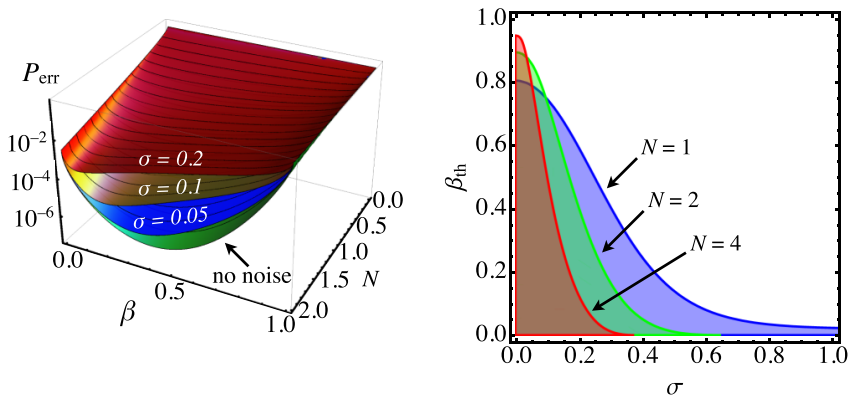

FIG. 2. (left) Error probability $P_{\text {err }}$ of the homodyne receiver in the presence of phase diffusion as a function of $\beta$ and $N$ for different values of the noise parameter $\sigma$. (right) Threshold value $\beta_{\text {th }}(\sigma)$ as a function of the noise parameter $\sigma$ for different values of $N$. The shaded regions refer to the couples of parameters $(\sigma, \beta)$ such that DDSs outperform coherent states.

where $D\left(\rho_{1}, \rho_{2}\right) \equiv \frac{1}{2}\left\|\rho_{1}-\rho_{2}\right\|_{1}$, with $\|A\|_{1}=\frac{1}{2} \operatorname{Tr}\left[\sqrt{A^{\dagger} A}\right]$, is the trace distance between the states $\rho_{1}$ and $\rho_{2}$.

In Fig. 3 we compare the Helstrom bound for the DSS with the error probability obtained by using the homodyne strategy (plots on the left side of Fig. 3). As in the case of the coherent states analyzed in Ref. [25], also when the binary information is encoded in DSSs the homodyne detection approaches the optimal measurement in the presence of phase diffusion. It is, however, worth noting that DSSs allow us to obtain a lower error probability and also to beat the Helstrom bound of the coherent-state case for small values of the noise parameter $\sigma$ (see the right side of Fig. 3).

\section{B. Effect of purity on discrimination}

The analysis we pursued in the previous sections focused on a pure seed state; namely, the squeezed vacuum $\left|\psi_{0}\right\rangle=$ $S(r)|0\rangle$. To take into account possible imperfections in the generation of squeezed states and losses in their propagation,
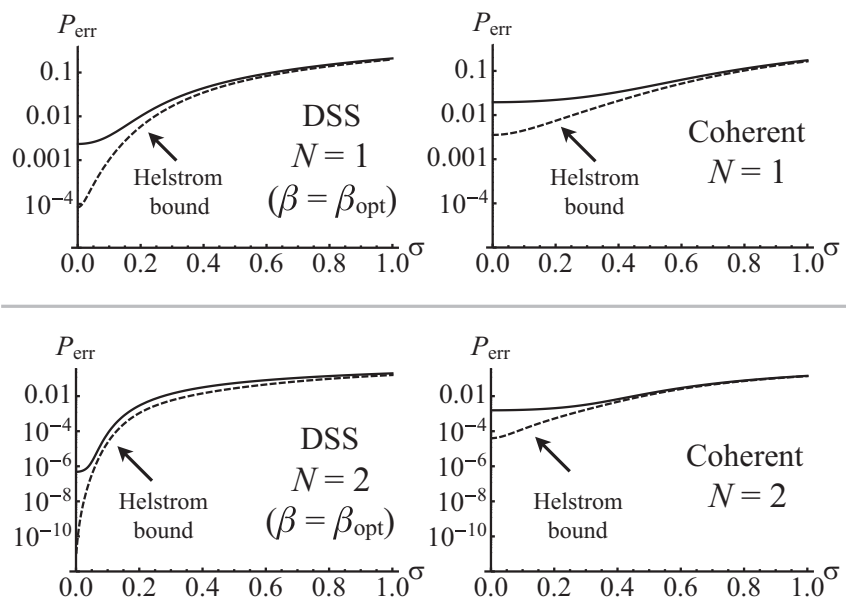

FIG. 3. Comparison between the error probability $P_{\text {err }}$ (solid lines) and the Helstrom bound (dashed lines) as a function of the noise parameter $\sigma$ for the DSS (left) and the coherent state (right) and for two different values of the energy: (top) $N=1$ and (bottom) $N=2$. Note that, in the presence of small values of $\sigma$, DSSs can beat the Helstrom bound of the corresponding coherent-state channel. For the DSS we used the optimal squeezing fraction $\beta_{\text {opt }}(N)$ given in Eq. (8).
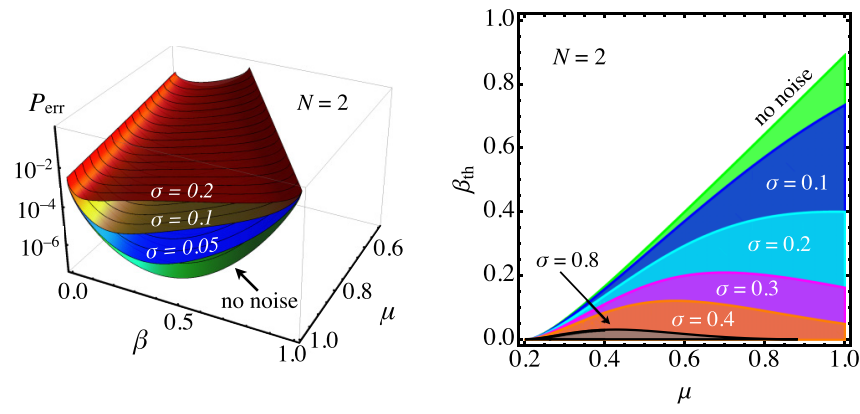

FIG. 4. (left) Error probability $P_{\text {err }}$ of the homodyne receiver as a function of $\beta$ and the purity $\mu$ of the seed state for different values of the noise parameter $\sigma$. We set $N=2$. (right) Threshold value $\beta_{\mathrm{th}}(\mu)$ as a function of the purity $\mu$ of the states for different values of the noise parameter $\sigma$. The shaded regions refer to the pairs of parameters $(\mu, \beta)$ for which DDSs outperform coherent states. Also here we set $N=2$. Note that $(1+2 N)^{-1} \leqslant \mu \leqslant 1$.

we extend our analysis to include the effect of a nonunit purity $\mu=\operatorname{Tr}\left[\rho_{0}^{2}\right]$ of the seed state which is now described by the density operator $\rho_{0}$. Without loss of generality, we can assume that, in this case, the seed is given by the squeezed thermal state [33]:

$$
\rho_{0}=S(r) v(\mu) S^{\dagger}(r),
$$

where $v(\mu)$ is a thermal state with average number of photons $N_{\text {th }}=(1-\mu) /(2 \mu)$. It is worth noting [34-36] that this is also equivalent to the scenario in which a pure squeezed vacuum state $\left|\psi_{0}\right\rangle=S(\tilde{r})|0\rangle$, corresponding to an initial squeezing of $10 \log _{10}\left(e^{2|\tilde{r}|}\right) \mathrm{dB}$, is sent through a channel with loss parameter $\eta$. In this case, the output state is given by Eq. (23) with

$$
\mu=\frac{e^{2 \tilde{r}}}{\left[\eta+(1-\eta) e^{2 \tilde{r}}\right]\left[1+\eta\left(e^{2 \tilde{r}}-1\right)\right]},
$$

and

$$
e^{2 r}=e^{\tilde{r}} \sqrt{\frac{1+\eta\left(e^{2 \tilde{r}}-1\right)}{\eta+(1-\eta) e^{2 \tilde{r}}}} .
$$

In this case we can still follow the discrimination strategy mentioned above, but now the total energy of the displaced input state $D( \pm \alpha) \rho_{0} D^{\dagger}( \pm \alpha)$ reads

$$
N=|\alpha|^{2}+N \beta+\frac{1-\mu}{2 \mu}(1+2 N \beta),
$$

where the squeezing fraction $\beta$ is the same as in Eq. (6). Note that $(1+2 N)^{-1} \leqslant \mu \leqslant 1$. In the left panel of Fig. 4 we plot the corresponding error probability as a function of $\beta$ and the purity $\mu$ for $N=2$ (analogous results can be found for other energies) for different values of the noise parameter $\sigma$ introduced in the previous section. As expected, the presence of a mixed seed state $(\mu<1)$ increases the error probability. Nevertheless, we can find again a threshold on the squeezing parameter below which this nonclassical resource enhances the discrimination with respect to the coherent state (for the same fixed energy). The threshold now depends also on the purity of the state, as shown in the right panel of Fig. 4.

It is interesting to note that there exists a threshold of $\sigma$ which depends on $N$ such that squeezing can help for given values of the purity (or of the channel losses), as shown in 

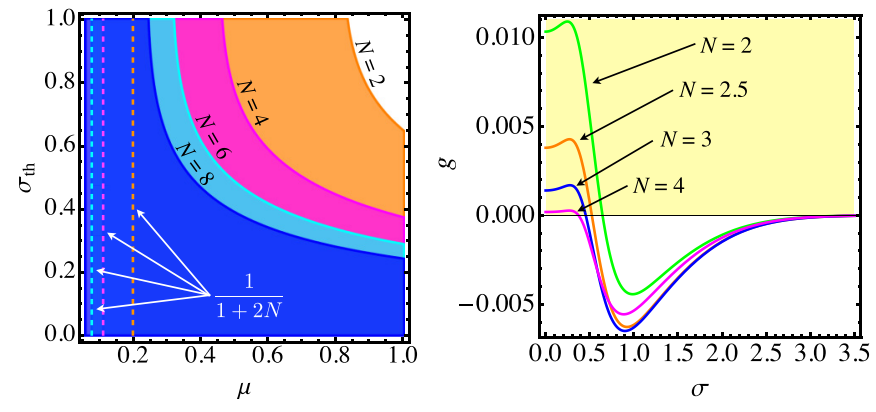

FIG. 5. (left) Plot of threshold $\sigma_{\text {th }}$ of the noise parameter as a function of purity $\mu$ and for different values of $N$. The vertical dashed lines refer to the lower limit of $\mu$ (see text for details). For $\sigma<\sigma_{\text {th }}$ squeezing is useful (of course below the corresponding $\beta_{\text {th }}$ ). (right) Plot of the function $g(N ; \sigma)$ in Eq. (28) as a function of $\sigma$ and for different values of $N$ : when its value is positive (shaded region) we have $P_{\text {err }}(\mu=1, \beta, N ; \sigma)<P_{\text {err, CS }}(N ; \sigma)$ in the limit $\beta \ll 1$. Note that, as $\mu$ decreases or, equivalently, as the thermal contribution gets larger, the threshold increases. Although the corresponding value of $\beta_{\text {th }}$ becomes extremely small, it does not vanish (see, for instance, the right panel of Fig. 4). See text for details.

the left panel of Fig. 5. The value of $\sigma_{\text {th }}$ can be obtained by expanding the error probability $P_{\text {err }}(\mu=1, \beta, N ; \sigma)$ for $\beta \ll 1$ in the case of DSSs. By considering the lowest order of $\beta$; namely, $\sqrt{\beta}$, we have

$$
P_{\mathrm{err}}(\mu=1, \beta, N ; \sigma) \approx P_{\mathrm{err}, \mathrm{CS}}(N ; \sigma)-g(N ; \sigma) \sqrt{\beta N},
$$

with

$$
g(N ; \sigma)=\int_{-\infty}^{+\infty} d \phi \frac{\exp \left(-\frac{\phi^{2}}{2 \sigma^{2}}\right)}{\sqrt{2 \pi \sigma^{2}}} \frac{e^{-2 N \cos ^{2} \phi} \cos (2 \phi) \cos \phi}{\sqrt{\pi}}
$$

and $\sigma_{\text {th }}$ is given by the maximum value of $\sigma$ such that $g(N ; \sigma)>0$ (see right panel of Fig. 5). Note that $P_{\text {err, CS }}(N ; \sigma) \equiv P_{\text {err }}(\mu=1,0, N ; \sigma)$ is the error probability in the presence of coherent states $(\beta=0)$.

\section{CONCLUSIONS}

We have investigated the role of squeezing in PSK quantum communication and shown that it represents a resource in the presence of phase diffusion and losses. In particular, we focused on binary encoding onto displaced squeezed states (DSSs) with coherent amplitudes having opposite phases. We have considered the ultimate limit to the error probability given by the the Helstrom bound, as well as a realistic scenario based on homodyne detection.

In the absence of noise there exists a threshold value $\beta_{\mathrm{th}}(N)$ on the squeezing fraction $\beta$ : below this threshold the Helstrom bound for DSSs is lower than that obtained by using coherent channels with the same energy, i.e., squeezing is a resource to improve discrimination. When phase diffusion is taken into account, the error probability unavoidably increases. However, we can find a threshold value $\beta_{\text {th }}(N, \sigma)$, now depending also on the phase-noise parameter $\sigma$, below which squeezing still provides enhanced discrimination.

Moreover, we have shown that a channel with DSSs encoding and homodyne detection approaches the optimality in the presence of phase noise, i.e., it may achieve an error probability close to the corresponding Helstrom bound. On the one hand, this confirms the findings obtained with coherent encoding [25]. On the other hand, we have found that the error probability obtained exploiting the squeezing may fall below the ultimate limit given by the Helstrom bound for coherent states.

Our results put squeezing forward as a resource for quantum communication channels in realistic conditions; namely, when phase-noise and losses occur during the generation and propagation of information carriers. They also pave the way for further developments in $M$-ary communication channels.
[1] H. P. Yuen, R. S. Kennedy, and M. Lax, Optimum testing of multiple hypotheses in quantum detection theory, IEEE Trans. Inf. Theory IT 21, 125 (1975).

[2] C. W. Helstrom, Quantum Detection and Estimation Theory (Academic Press, New York, 1976).

[3] J. A. Bergou, U. Herzog, and M. Hillery, Discrimination of quantum states, in Quantum State Estimation, edited by M. Paris and J. Řeháček, Lecture Notes in Physics (Springer, Berlin, Heidelberg, 2004), Vol. 649, p. 415.

[4] A. Chefles, Quantum states: Discrimination and classical information transmission. A review of experimental progress, in Quantum State Estimation, edited by M. Paris and J. Řeháček Lecture Notes in Physics (Springer, Berlin, Heidelberg, 2004), Vol. 649, p. 465.

[5] J. A. Bergou, Discrimination of quantum states, J. Mod. Opt. 57, 160 (2010).

[6] I. D. Ivanovic, How to differentiate between non-orthogonal states, Phys. Lett. A 123, 257 (1987).
[7] D. Dieks, Overlap and distinguishability of quantum states, Phys. Lett. A 126, 303 (1988).

[8] A. Peres, How to differentiate between non-orthogonal states, Phys. Lett. A 128, 19 (1988).

[9] N. Tomassoni and M. G. A. Paris, Quantum binary channels with mixed states, Phys. Lett. A 373, 61 (2008).

[10] G. Cariolaro, Quantum Communication (Springer, Heidelberg, 2015).

[11] K. Günthner, I. Khan, D. Elser, B. Stiller, Ö. Bayraktar, C. R. Müller, K. Saucke, D. Tröndle, F. Heine, S. Seel, P. Greulich, H. Zech, B. Gütlich, S. Philipp-May, C. Marquardt, and G. Leuchs, Quantum-limited measurements of optical signals from a geostationary satellite, Optica 4, 611 (2017).

[12] E. Diamanti, H.-K. Lo, B. Qi, and Z. Yuan, Practical challenges in quantum key distribution, npj Quantum Inf. 2, 16025 (2016).

[13] F. E. Becerra, J. Fan, G. Baumgartner, J. Goldhar, J. T. Kosloski, and A. Migdall, Experimental demonstration of a receiver 
beating the standard quantum limit for multiple nonorthogonal state discrimination, Nat. Photon. 7, 147 (2013).

[14] C. Wittmann, U. L. Andersen, M. Takeoka, D. Sych, and G. Leuchs, Demonstration of Coherent-State Discrimination Using a Displacement-Controlled Photon-Number-Resolving Detector, Phys. Rev. Lett. 104, 100505 (2010).

[15] C. R. Müller, M. A. Usuga, C. Wittmann, M. Takeoka, C. Marquardt, U. L. Andersen, and G. Leuchs, Quadrature phase shift keying coherent state discrimination via a hybrid receiver, New J. Phys. 14, 083009 (2012).

[16] F. E. Becerra, J. Fan, G. Baumgartner, S. V. Polyakov, J. Goldhar, J. T. Kosloski, and A. Migdall, $M$-ary-state phase-shift-keying discrimination below the homodyne limit, Phys. Rev. A 84, 062324 (2011).

[17] R. Nair, B. J. Yen, S. Guha, J. H. Shapiro, and S. Pirandola, Symmetric $M$-ary phase discrimination using quantum-optical probe states, Phys. Rev. A 86, 022306 (2012).

[18] M. G. A. Paris, Nearly ideal binary communication in squeezed channels, Phys. Rev. A 64, 014304 (2001).

[19] S. Izumi, M. Takeoka, K. Ema, and M. Sasaki, Quantum receivers with squeezing and photon-number-resolving detectors for $M$-ary coherent state discrimination, Phys. Rev. A 87, 042328 (2013).

[20] C.-W. Lau, V. A. Vilnrotter, S. Dolinar, J. M. Geremia, and H. Mabuchi, Binary quantum receiver concept demonstration, Proc. SPIE 6105, 61050J (2006).

[21] R. S. Kennedy, A near-optimum receiver for the binary coherent state quantum channel, MIT Res. Lab. Electron. Quart. Progr. Rep. 108, 219 (1973).

[22] S. J. Dolinar Jr., An optimum receiver for the binary coherent state quantum channel, MIT Res. Lab. Electron. Quart. Progr. Rep. 111, 115 (1973).

[23] M. Bina, A. Allevi, M. Bondani, and S. Olivares, Phase-reference monitoring in coherent-state discrimination assisted by a photonnumber resolving detector, Sci. Rep. 6, 26025 (2016).

[24] L. G. Kazovsky, G. Kalogerakis, and W. T. Shaw, Homodyne phase-shift-keying systems: Past challenges and future opportunities, J. Lightwave Technol. 24, 4876 (2006).
[25] S. Olivares, S. Cialdi, F. Castelli, and M. G. A. Paris, Homodyne detection as a near-optimum receiver for phase-shift-keyed binary communication in the presence of phase diffusion, Phys. Rev. A 87, 050303(R) (2013).

[26] V. C. Usenko and R. Filip, Squeezed-state quantum key distribution upon imperfect reconciliation, New J. Phys. 13, 113007 (2011).

[27] S. Izumi, M. Takeoka, M. Fujiwara, N. Dalla Pozza, A. Assalini, K. Ema, and M. Sasaki, Displacement receiver for phase-shift-keyed coherent states, Phys. Rev. A 86, 042328 (2012).

[28] S. Izumi, M. Takeoka, K. Wakui, M. Fujiwara, K. Ema, and M. Sasaki, Optical phase estimation via coherent state and displaced photon counting, Phys. Rev. A 94, 033842 (2016).

[29] M. Bina, A. Allevi, M. Bondani, and S. Olivares, Homodyne-like detection for coherent state-discrimination in the presence of phase noise, Opt. Express 25, 10685 (2017).

[30] H. A. Bachor and T. Ralph, A Guide to Experiments in Quantum Optics (Wiley-VCH Verlag, Weinheim, 2004).

[31] M. G. Genoni, S. Olivares, and M. G. A. Paris, Optical Phase Estimation in the Presence of Phase Diffusion, Phys. Rev. Lett. 106, 153603 (2011).

[32] C. Gardiner and P. Zoller, Quantum Noise (Springer, Berlin, 2004).

[33] S. Olivares, Quantum optics in the phase space, Eur. Phys. J. Spec. Top. 203, 3 (2012).

[34] S. Cialdi, C. Porto, D. Cipriani, S. Olivares, and M. G. A. Paris, Full quantum state reconstruction of symmetric twomode squeezed thermal states via spectral homodyne detection and a state-balancing detector, Phys. Rev. A 93, 043805 (2016).

[35] V. D'Auria, S. Fornaro, A. Porzio, S. Solimeno, S. Olivares, and M. G. A. Paris, Full Characterization of Gaussian Bipartite Entangled States by a Single Homodyne Detector, Phys. Rev. Lett. 102, 020502 (2009).

[36] S. Olivares and M. G. A. Paris, Binary optical communication in single-mode and entangled quantum noisy channels, J. Opt. B: Quantum Semiclassical Opt. 6, 69 (2004). 